\title{
APPENDIX IV. INTERSTITIAL WATER CHEMISTRY: DEEP SEA DRILLING PROJECT, LEGS 21 AND 22
}

\author{
Bob J. Presley, John Trefry, Dan Armstrong, and Mike Nuzzo, \\ Department of Oceanography, Texas A\&M University, College Station, Texas
}

\section{INTRODUCTION AND PROCEDURES}

Leg 21 of the Deep Sea Drilling Project consisted of operations around Australia in some of the marginal basins of the Pacific. Leg 22 was also largely a southern hemisphere operation, but followed Ninetyeast Ridge to almost $10^{\circ} \mathrm{N}$ latitude before turning west to end in Colombo, Ceylon. These legs were conducted in late 1971 and early 1972 , but the pore waters sent to Texas A\&M were not analyzed until June 1973. This long storage could have adversely affected the pore water chemistry, especially for such constituents as silicon and ammonia, but we have no evidence that such is the case, and no unexpected values or trends that might be attributed to storage are evident. We intend to compare our data with that of the Woods Hole group when their report is received, and this should shed some light on storage effects that will be useful to future investigations.

The samples we received consisted of $2-3 \mathrm{ml}$ aliquots of membrane-filtered water that had been heat sealed into short sections of polyethylene tubing. They were kept refrigerated at all times between collection and analysis, except for transit by air mail.

The analytical procedures used are essentially the same as those described in an earlier DSDP report (Presley, 1971). We have, as usual, analyzed all samples for major cations and are including the Leg 21 data in this report. The Woods Hole group is reporting major cation data on the Leg 22 samples, as they have on all previous legs; therefore, our data have been omitted from the report.

\section{RESULTS AND DISCUSSION}

All samples have, as usual, been analyzed for chloride. This analysis is done using only $0.1 \mathrm{ml}$ of water, and only a few duplicates are run due to the small sample size and time requirements. Duplicates, or repeat runs on suspicious values, seldom disagree with the first value determined by more than 0.1 parts per thousand, thus the values given in Tables 1 and 2 are thought to be reliable for the purpose intended.

Samples from previous legs have almost always given $\mathrm{Cl}$ values within a few percent of that of present-day seawater, except where evaporite deposits are present, or where continental fresh water has had an influence. There have been, however, a few samples from several previous legs that were difficult to explain by either of these mechanisms. In some cases, where only a single isolated value is involved, sample contamination during coring or storage must be suspected. This might be true of Samples 206-11-3 and 218-15-1 in the present group. In other cases, however, unmistakable trends with depth below the sea floor can be seen, and these must have considerable geochemical significance, although the values are difficult to explain at this time. An example of this is the low $\mathrm{Cl}$ values found on Legs 16 and 19 and the high values found in some of the present samples (see also the Woods Hole report, Chapter 31). In any case, some reevaluation of the chlorinity or salinity of all the DSDP pore waters should be made at some future date with a view towards explaining the anomalous values.

One aid in explaining salinity changes is the bromide concentration, as we have pointed out in previous leg reports. In the present group of samples, for example, the anomalous $\mathrm{Cl}$ value at Site 206 is matched almost exactly by an anomalous $\mathrm{Br}$ value so as to leave the $\mathrm{Cl} / \mathrm{Br}$ ratio unchanged. At Site 218 , on the other hand, the $\mathrm{Br}$ seems to be impoverished by about $20 \%$. Thus, it is easy to explain Sample 203-11-3 as representing a simple dilution with fresh water, but it is not so easy to explain Sample 218-11-2, unless, of course, an error has been made in one of the measurements.

In the same way, the almost $13 \%$ chlorinity increase at Site 216 is accompanied by a corresponding $\mathrm{Br}$ increase so the ratio changes by only a little over $1 \%$, but at Site 214 the $\mathrm{Br}$ increase is much larger than that of $\mathrm{Cl}$ so the ratio goes down by about 13\%. A similar, but less clear-cut, case occurs at Site 210 . The $\mathrm{Br}$ and $\mathrm{Cl}$ values can, then, aid in interpreting the geological history of a site with respect to influences by evaporite deposition and leaching.

The major cation concentrations at the Leg 21 sites show most of the phenomenon we have been observing on the DSDP samples since Leg 4 . That is, either little change with depth of burial, or pronounced trends of decreasing $\mathrm{Mg}$ and $\mathrm{K}$ and increasing $\mathrm{Ca}$. The factors which determine which trend occurs have still not been worked out. At Site 210 we also see a rather drastic decrease in $\mathrm{Ca}$ concentration in the Pleistocene sediments. This is undoubtedly due to an increase in carbonate alkalinity, as explained by Presley and Kaplan (1968), but the decrease may have been intensified by precipitation during storage as $\mathrm{CO}_{2}$ was lost by outgassing. The $\mathrm{Ca}$ increases at Sites 204 and 210 are by more than $350 \%$, but even more drastic increases were found in some of the Leg 22 samples (see also Chapter 31). These large changes in Ca concentration can only be due to rather extensive diagenetic changes in the solid phases of the sediment.

Diagenetic changes are also evident from the silicon measurements because there are, as usual, large differences in concentration at different depths at most sites. It is difficult to see how these concentration gradients can be 
TABLE 1

Selected Major and Minor Constituents, Interstitial Water, Leg 21

\begin{tabular}{|c|c|c|c|c|c|c|c|c|c|c|c|c|c|}
\hline Samplea & $\begin{array}{l}\text { Depthb } \\
\text { (m) }\end{array}$ & $\begin{array}{l}\text { Age and Description } \\
\text { of Sediment }^{\mathrm{c}}\end{array}$ & $\underset{(\mathrm{g} / \mathrm{kg})}{\mathrm{Cl}}$ & $\underset{(\mathrm{mg} / \mathrm{kg})}{\mathrm{Br}}$ & $\mathrm{Cl} / \mathrm{Br}$ & $\begin{array}{l}\mathrm{Na} \\
(\mathrm{g} / \mathrm{kg})\end{array}$ & $\underset{(\mathrm{mg} / \mathrm{kg})}{\mathrm{K}}$ & $\underset{(\mathrm{mg} / \mathrm{kg})}{\mathrm{Ca}}$ & $\underset{(\mathrm{g} / \mathrm{kg})}{\mathrm{Mg}}$ & $\underset{(\mathrm{mg} / \mathrm{kg})}{\mathrm{Si}}$ & $\underset{(\mathrm{mg} / \mathrm{kg})}{\mathrm{Mn}}$ & $\underset{(\mathrm{mg} / \mathrm{kg})}{\mathrm{Li}}$ & $\underset{(\mathrm{mg} / \mathrm{kg})}{\mathrm{NH}_{3}}$ \\
\hline 203-1-2 & 2 & Pleistocene; ashy $\mathrm{CaCO}_{3}$ voze & 19.8 & 67 & 294 & 10.9 & 423 & 416 & 1.37 & 11.3 & 2.79 & 0.18 & 0.7 \\
\hline 203-3-6 & 117 & Pleistocene; ashy $\mathrm{CaCO}_{3}$ ooze & 19.8 & 68 & 289 & 11.3 & 409 & 425 & 1.36 & 12.3 & 7.37 & 0.20 & $<0.1$ \\
\hline 204-1-5 & 6 & Quaternary; dark brown silty clay & 20.1 & 68 & 296 & 11.2 & 433 & 444 & 1.34 & 15.4 & $<0.01$ & 0.19 & 1.5 \\
\hline $204-4-4$ & 89 & Eocene (?); dark brown clay, $\mathrm{Fe}_{2} \mathrm{O}_{3}$ & 21.1 & 71 & 297 & 9.8 & 437 & 1470 & 0.97 & 6.8 & 0.02 & 0.18 & $<0.1$ \\
\hline 205-3-6 & 27 & $\begin{array}{l}\text { Late } \mathrm{Miocene} ; \mathrm{Fe}_{2} \mathrm{O}_{3} \text { clay, thin } \\
\quad \mathrm{CaCO}_{3} \text { beds }\end{array}$ & 20.2 & 68 & 295 & 11.1 & 451 & 498 & 1.32 & 10.9 & $<0.01$ & 0.15 & $<0.1$ \\
\hline $205-10-2$ & 86 & Late Miocene; $\mathrm{CaCO}_{3}$ ooze, ash & 20.1 & 69 & 289 & 11.0 & 438 & 745 & 1.05 & 14.3 & 3.42 & 0.07 & 2.3 \\
\hline $205-20-2$ & 181 & Middle Miocene; vitric tuff, $\mathrm{CaCO}_{3}$ & 20.0 & 69 & 293 & 11.1 & 422 & 871 & 0.89 & 15.2 & 3.15 & 0.10 & 1.4 \\
\hline 206-1-3 & 2 & $\begin{array}{l}\text { Pleistocene; } \mathrm{CaCO}_{3} \text { ooze, glass } \\
\text { shards }\end{array}$ & 20.3 & 68 & 297 & 11.0 & 451 & 408 & 1.26 & 13.9 & 0.82 & 0.18 & 1.0 \\
\hline 206-11-3 & 95 & Pleistocene; $\mathrm{CaCO}_{3}$ ooze & 16.6 & 57 & 289 & 9.2 & 367 & 369 & 0.93 & 21.3 & 0.04 & 0.13 & 5.2 \\
\hline $206-24-6$ & 219 & $\begin{array}{l}\text { Late Miocene; } \mathrm{CaCO}_{3} \text { ooze, } \\
\qquad \mathrm{SiO}_{2} \text { beds }\end{array}$ & 19.7 & 69 & 284 & 10.8 & 409 & 571 & 0.98 & 26.2 & 0.04 & 0.11 & 7.6 \\
\hline $207-5-5$ & 46 & Pliocene; $\mathrm{CaCO}_{3}$ ooze, glass shards & 20.1 & 70 & 287 & 10.8 & 421 & 454 & 1.31 & 7.6 & 0.05 & 0.23 & 1.0 \\
\hline $207 \mathrm{~A}-7-5$ & 109 & Miocene; $\mathrm{CaCO}_{3}$ ooze, $\mathrm{SiO}_{2}$ beds & 20.0 & 69 & 288 & 10.7 & 387 & 782 & 1.24 & 8.6 & 0.02 & 0.33 & 1.6 \\
\hline $207 \mathrm{~A}-21-4$ & 243 & Eocene; $\mathrm{CaCO}_{3}$ ooze, $\mathrm{SiO}_{2}$ beds & 20.0 & 70 & 284 & 10.8 & 370 & 571 & 1.13 & 25.5 & 0.07 & 0.50 & 3.0 \\
\hline $\begin{array}{l}208-4-6 \\
208-14-6\end{array}$ & $\begin{array}{r}44 \\
166\end{array}$ & Pliocene; $\mathrm{CaCO}_{3}$ ooze, pyrite & 20.1 & 69 & 290 & 10.9 & 403 & 429 & 1.27 & 4.8 & 0.04 & 0.18 & 0.3 \\
\hline 208-14-6 & 166 & Miocene; $\mathrm{CaCO}_{3}$ ooze, pyrite & 20.2 & 70 & 286 & 10.9 & 361 & 494 & 1.15 & 6.8 & 0.05 & 0.24 & 2.3 \\
\hline $\begin{array}{l}209-2-6 \\
209-6-6\end{array}$ & $\begin{array}{l}18 \\
54\end{array}$ & Pleistocene; $\mathrm{CaCO}_{3}$ ooze & 19.8 & 70 & 284 & 10.7 & 409 & 444 & 1.24 & 4.8 & 0.32 & 0.22 & 1.7 \\
\hline $209-6-6$ & 54 & Miocene; $\mathrm{CaCO}_{3}$ ooze & 20.2 & 70 & 290 & 10.9 & 390 & 518 & 1.26 & 5.1 & 0.09 & 0.30 & 0.6 \\
\hline 210-1-6 & 9 & Pleistocene; silty clay, $\mathrm{CaCO}_{3}$ beds & 19.5 & 68 & 287 & 10.7 & 438 & 327 & 1.11 & 9.0 & 3.60 & 0.11 & 7.6 \\
\hline $210-10-4$ & 87 & Pleistocene; silty clay, $\mathrm{CaCO}_{3}$ beds & 19.8 & 71 & 278 & 10.8 & 330 & 137 & 0.88 & 7.9 & 0.21 & 0.11 & 12.6 \\
\hline $210-27-5$ & 411 & Miocene; silty clay, $\mathrm{CaCO}_{3}$ beds & 20.2 & 75 & 269 & - & 116 & 1486 & 0.28 & 2.6 & 1.70 & 0.41 & 18.2 \\
\hline \multicolumn{3}{|c|}{ Average seawater } & 19.4 & 67 & 290 & 10.8 & 387 & 413 & 1.29 & $0-5$ & $<0.01$ & 0.17 & $<0.1$ \\
\hline
\end{tabular}

aHole-core-section.

bDepth below sea floor.

cFrom p̀reliminary hole summaries. 
TABLE 2

Selected Major and Minor Constituents, Interstitial Water, Leg 22

\begin{tabular}{|c|c|c|c|c|c|c|c|c|c|}
\hline Samplea & $\begin{array}{l}\text { Depthb } \\
\text { (m) }\end{array}$ & $\begin{array}{l}\text { Age and Description } \\
\text { of Sediment } \mathrm{c}^{\mathrm{c}}\end{array}$ & $\underset{(\mathrm{g} / \mathrm{kg})}{\mathrm{Cl}}$ & $\underset{(\mathrm{mg} / \mathrm{kg})}{\mathrm{Br}}$ & $\mathrm{Cl} / \mathrm{Br}$ & $\underset{(\mathrm{mg} / \mathrm{kg})}{\mathrm{Si}}$ & $\underset{(\mathrm{mg} / \mathrm{kg})}{\mathrm{Mn}}$ & $\underset{(\mu \mathrm{g} / \mathrm{kg})}{\mathrm{Li}}$ & $\underset{(\mathrm{mg} / \mathrm{kg})}{\mathrm{NH}_{3}}$ \\
\hline $\begin{array}{l}211-1-3 \\
211-5-5\end{array}$ & $\begin{array}{r}5 \\
141\end{array}$ & $\begin{array}{l}\text { Quaternary; clay-rich rad ooze, ash } \\
\text { Pliocene; clay-rich rad ooze }\end{array}$ & $\begin{array}{l}19.4 \\
20.0\end{array}$ & $\begin{array}{l}67 \\
71\end{array}$ & $\begin{array}{l}289 \\
281\end{array}$ & $\begin{array}{l}19.4 \\
22.9\end{array}$ & $\begin{array}{r}15.5 \\
5.9\end{array}$ & $\begin{array}{l}187 \\
170\end{array}$ & $\begin{array}{r}<0.1 \\
7.4\end{array}$ \\
\hline $212-2-3$ & 14 & $\begin{array}{l}\text { Pliocene; interbedded nanno ooze } \\
\text { and clay }\end{array}$ & 20.1 & 69 & 290 & 5.4 & 0.5 & 230 & 0.5 \\
\hline $212-8-3$ & 131 & Miocene; nanno ooze and chalk & 20.0 & 71 & 283 & 5.5 & 0.9 & 295 & 5.0 \\
\hline $\begin{array}{l}212-11-3 \\
212-15-3\end{array}$ & $\begin{array}{l}198 \\
293\end{array}$ & $\begin{array}{l}\text { Miocene; nanno ooze and chalk } \\
\text { Miocene; zeolitic claystone }\end{array}$ & $\begin{array}{l}20.3 \\
19.5\end{array}$ & $\begin{array}{l}72 \\
69\end{array}$ & $\begin{array}{l}282 \\
280\end{array}$ & $\begin{array}{l}8.0 \\
7.6\end{array}$ & $\begin{array}{l}0.6 \\
1.7\end{array}$ & $\begin{array}{l}370 \\
408\end{array}$ & $\begin{array}{l}3.2 \\
4.4\end{array}$ \\
\hline $\begin{array}{l}213-2-5 \\
213-5-5 \\
213-9-6 \\
213-13-6 \\
213-16-4\end{array}$ & $\begin{array}{r}17 \\
45 \\
85 \\
123 \\
148\end{array}$ & $\begin{array}{l}\text { Pliocene; clay-bearing } \mathrm{SiO}_{2} \text { ooze } \\
\text { Pliocene; clay-bearing } \mathrm{SiO}_{2} \text { ooze } \\
\text { Miocene; zeolitic clay, } \mathrm{Mn}-\mathrm{Fe} \text { oxide } \\
\text { Eocene; zeolitic clay, } \mathrm{Mn}-\mathrm{Fe} \text { oxide } \\
\text { Paleocene; nanno ooze, } \mathrm{Mn}-\mathrm{Fe} \\
\quad \text { oxide }\end{array}$ & $\begin{array}{l}20.0 \\
19.8 \\
19.8 \\
19.6 \\
20.1\end{array}$ & $\begin{array}{l}69 \\
69 \\
69 \\
69 \\
72\end{array}$ & $\begin{array}{l}291 \\
288 \\
286 \\
285 \\
280\end{array}$ & $\begin{array}{r}22.9 \\
23.4 \\
21.8 \\
5.2 \\
4.1\end{array}$ & $\begin{array}{r}13.3 \\
13.4 \\
12.0 \\
0.1 \\
0.1\end{array}$ & $\begin{array}{l}230 \\
253 \\
253 \\
227 \\
190\end{array}$ & $\begin{array}{r}<0.1 \\
<0.1 \\
8.3 \\
<0.1 \\
<0.1\end{array}$ \\
\hline $\begin{array}{l}214-1-5 \\
214-9-5 \\
214-19-5 \\
214-31-5\end{array}$ & $\begin{array}{r}8 \\
84 \\
179 \\
293\end{array}$ & $\begin{array}{l}\text { Pleistocene; } \mathrm{CaCO}_{3} \text { ooze } \\
\text { Pliocene; } \mathrm{CaCO}_{3} \text { ooze } \\
\text { Miocene; } \mathrm{CaCO}_{3} \text { ooze } \\
\text { Eocene; } \mathrm{CaCO}_{3} \text { ooze }\end{array}$ & $\begin{array}{l}19.9 \\
19.9 \\
20.1 \\
20.4\end{array}$ & $\begin{array}{l}69 \\
70 \\
73 \\
80\end{array}$ & $\begin{array}{l}289 \\
284 \\
276 \\
255\end{array}$ & $\begin{array}{l}15.9 \\
19.3 \\
21.9 \\
10.5\end{array}$ & $\begin{array}{l}0.1 \\
0.1 \\
0.2 \\
0.6\end{array}$ & $\begin{array}{r}167 \\
92 \\
92 \\
159\end{array}$ & $\begin{array}{l}0.2 \\
1.5 \\
3.6 \\
5.1\end{array}$ \\
\hline $\begin{array}{l}215-1-5 \\
215-8-3 \\
215-10-2 \\
215-17-1\end{array}$ & $\begin{array}{r}8 \\
69 \\
86 \\
150\end{array}$ & $\begin{array}{l}\text { Quaternary; } \mathrm{SiO}_{2} \text { ooze } \\
\text { Miocene; rad-bearing clay } \\
\text { Eocene; clay-rich nanno ooze } \\
\text { Paleocene; as above with } \mathrm{Fe}_{2} \mathrm{O}_{3}\end{array}$ & $\begin{array}{l}20.0 \\
20.0 \\
19.6 \\
19.6\end{array}$ & $\begin{array}{l}70 \\
71 \\
69 \\
69\end{array}$ & $\begin{array}{l}285 \\
283 \\
284 \\
283\end{array}$ & $\begin{array}{r}21.5 \\
20.6 \\
20.8 \\
9.6\end{array}$ & $\begin{array}{r}6.8 \\
5.3 \\
<0.1 \\
-\end{array}$ & $\begin{array}{c}171 \\
195 \\
167 \\
-\end{array}$ & $\begin{array}{r}<0.1 \\
0.8 \\
0.4 \\
-\end{array}$ \\
\hline $\begin{array}{l}216-1-6 \\
216-5-5 \\
216-14-3 \\
216-25-4\end{array}$ & $\begin{array}{r}9 \\
166 \\
149 \\
354\end{array}$ & $\begin{array}{l}\text { Pleistocene; } \mathrm{CaCO}_{3} \text { ooze, ash } \\
\text { Miocene; } \mathrm{CaCO}_{3} \text { ooze } \\
\text { Oligocene; Nanno chalk } \\
\text { Cretaceous; chalk, ash, feldspar, } \\
\quad \text { clay }\end{array}$ & $\begin{array}{l}19.6 \\
20.4 \\
20.2 \\
22.1\end{array}$ & $\begin{array}{l}68 \\
73 \\
75 \\
77\end{array}$ & $\begin{array}{l}289 \\
279 \\
281 \\
285\end{array}$ & $\begin{array}{r}15.1 \\
26.5 \\
27.4 \\
2.9\end{array}$ & $\begin{array}{l}0.4 \\
0.2 \\
0.2 \\
0.4\end{array}$ & $\begin{array}{l}162 \\
136 \\
180 \\
204\end{array}$ & $\begin{array}{l}0.4 \\
1.5 \\
3.3 \\
6.7\end{array}$ \\
\hline $217-1-6$ & 9 & Quaternary; $\mathrm{CaCO}_{3}$ ooze with clay & 19.6 & 69 & 286 & 19.7 & 1.9 & 161 & $<0.1$ \\
\hline $217-4-4$ & 122 & $\begin{array}{l}\text { Miocene; } \mathrm{CaCO}_{3} \text { ooze, } \mathrm{SiO}_{2} \text { ooze, } \\
\text { clay }\end{array}$ & 20.3 & 71 & 286 & 25.0 & 0.2 & 513 & 2.3 \\
\hline $217-9-6$ & 315 & $\begin{array}{l}\text { Oligocene; clay-bearing nanno } \\
\text { chalk }\end{array}$ & 21.6 & 77 & 281 & 33.1 & 0.4 & 750 & 4.1 \\
\hline $218-2-4$ & 10 & $\begin{array}{l}\text { Pleistocene; nanno-bearing } \\
\text { clayey silt }\end{array}$ & 19.9 & 69 & 289 & 16.1 & 1.9 & 105 & 7.1 \\
\hline $218-5-2$ & 73 & $\begin{array}{l}\text { Pleistocene; nanno-bearing } \\
\text { clayey silt }\end{array}$ & 20.2 & 72 & 280 & 12.3 & 0.7 & 117 & 8.5 \\
\hline $218-8-3$ & 189 & $\begin{array}{l}\text { Pleistocene; nanno-bearing } \\
\text { silty clay }\end{array}$ & 20.2 & 70 & 288 & 11.8 & 0.3 & 137 & 21.8 \\
\hline $218-11-2$ & 301 & $\begin{array}{l}\text { Pliocene; } \mathrm{CaCO}_{3} \text { ooze, silt and } \\
\text { clay }\end{array}$ & 20.2 & 71 & 285 & 4.6 & 0.6 & 405 & 11.7 \\
\hline $218-15-1$ & 450 & $\begin{array}{l}\text { Miocene; sandy silt, calcite, } \\
\text { heavies }\end{array}$ & 11.8 & 34 & 351 & 2.1 & 0.1 & 57 & 10.2 \\
\hline $\begin{array}{l}218-21-3 \\
218-25-2\end{array}$ & $\begin{array}{l}540 \\
690\end{array}$ & $\begin{array}{l}\text { Miocene; laminated sandy silt } \\
\text { Miocene; silt and chalk }\end{array}$ & $\begin{array}{l}20.8 \\
20.9\end{array}$ & $\begin{array}{l}72 \\
73\end{array}$ & $\begin{array}{l}287 \\
286\end{array}$ & $\begin{array}{l}4.2 \\
3.3\end{array}$ & $\begin{array}{l}0.3 \\
0.5\end{array}$ & $\begin{array}{l}128 \\
158\end{array}$ & $\begin{array}{l}18.8 \\
18.6\end{array}$ \\
\hline \multicolumn{3}{|c|}{ Average seawater } & 19.4 & 67 & 290 & $0-5$ & $<0.01$ & 170 & $<0.1$ \\
\hline
\end{tabular}

aHole-core-section

bDepth below sea floor.

cFrom preliminary hole summaries. 
maintained unless continuous reactions are occurring.

Manganese concentrations also show their usual wide variation, with the upper samples at Site 213 being among the highest yet found during the DSDP, yet many samples are near or below our detection limit. There is again no obvious relationship to lithology. Some of the samples where Fe-Mn oxides were conspicuous in the solids gave high dissolved $\mathrm{Mn}$, but some gave low values.

Lithium concentrations have increased sharply with depth at many sites on previous legs, and this is shown well at Site 217. We still have no explanation for the phenomenon, however, nor any explanation for the low $\mathrm{Li}$ values found at a few locations.

Ammonia would seem to be more likely to change with the long storage these samples were subjected to than the other components, yet there is no indication this has happened. The high values were found at Sites 210 and 218 , locations where the $\mathrm{Ca}$ data indicate active biological activity. Sulfate concentrations would offer the best confirmation of this supposition, but we have no sulfate data at this time.

\section{ACKNOWLEDGMENTS}

This work was supported in part by NSF grant 6A-20715.

\section{REFERENCES}

Presley, B. J., 1971. Techniques for analyzing interstitial water samples. Part I: Determination of selected minor and major inorganic constituents. In Winterer, E. L. et al., Initial Reports of the Deep Sea Drilling Project, Volume VII: Washington (U.S. Government Printing Office), $\mathrm{p}$.

Presley, B. J. and Kaplan, I. R., 1968. Changes in dissolved sulfate, calcium and carbonate from interstitial water of nearshore sediments: Geochim. Cosmochim. Acta, v. 32 , p. 1037. 